Computer Science and Systems Analysis

Computer Science and Systems Analysis

Technical Reports

\title{
Interface-based Programming Assignments and Automatic Grading of Java Programs
}

Michael T. Helmick

Miami University

This paper is posted at Scholarly Commons at Miami University.

http://sc.lib.muohio.edu/csa_techreports/2 


\title{
Interface-based Programming Assignments and Automatic Grading of Java Programs
}

\author{
Michael T Helmick \\ Miami University \\ Department of Computer Science and Systems Analysis \\ Oxford, Ohio USA \\ mike.helmick@muohio.edu
}

\begin{abstract}
AutoGrader is a framework developed at Miami University for the automatic grading of student programming assignments written in the Java programming language. AutoGrader leverages the abstract concept of interfaces, brought out by the Java interface language construct, in both the assignment and grading of programming assignments. The use of interfaces reinforces the role of procedural abstraction in object-oriented programming and allows for a common API to all student code. This common API then enables automatic grading of program functionality. AutoGrader provides a simple instructor API and enables the automatic testing of student code through the Java language features of interfaces and reflection ${ }^{1}$. AutoGrader also supports static code analysis using PMD [4] to detect possible bugs, dead code, suboptimal, and overcomplicated code. While AutoGrader is written in and only handles Java programs, this style of automated grading is adaptable to any language that supports (or can mimic) named interfaces and/or abstract functions and that also supports runtime reflection.
\end{abstract}

\section{INTRODUCTION}

Automatic grading of student programs has been of interest to computer science educators for some time [8] and continues to gain attention today $[7,5,9,12,14,10]$. There have been various approaches with most leaning towards analyzing the output of the entire program with some mention of techniques and tools such as JUnit [16]. However, there seems to be no established consensus on the best way to automatically grade student code.

We take the position that there are two tasks involved in grading of student assignments:

\section{Functional testing}

2. Evaluation of design and style

${ }^{1}$ The Java reflection API allows the runtime system to be inspected and manipulated dynamically. http://java.sun. com/docs/books/tutorial/reflect/index.html

Permission to make digital or hard copies of all or part of this work for personal or classroom use is granted without fee provided that copies are not made or distributed for profit or commercial advantage and that copies bear this notice and the full citation on the first page. To copy otherwise, to republish, to post on servers or to redistribute to lists, requires prior specific permission and/or a fee.

Copyright 2006 Miami University Technical Report: MU-SEAS-CSA2006-002
Preliminary analysis of Java coding style can be handled automatically using either the open source Checkstyle [1] or PMD [4] packages (AutoGrader is integrated with PMD). While it might be possible to handle the evaluation of program design in an automated fashion, we feel that this task is best left to an experienced instructor or teaching assistant. There is a large body of program design knowledge that comes from experience, and this experience can not easily be programmed into an automated system.

Functional testing, on the other hand, is a task that produces a boolean answer. A program either works and provides the correct answer based on predetermined inputs, or it does not. Since software systems tend to be built in layers, we can further sub-divide assignments and target specific functionalities for testing. Our goal in automatically testing student code is two-fold: (1) to adequately exercise student code; and (2) to segment the tests such that pieces of functionality are isolated and tested as independently as possible. This not only makes automatic assessment easier, it is also a valuable teaching technique for instruction of software construction.

We have introduced a pedagogy for our data structures course that enables and utilizes automatic grading for programming assignment functionality. Interface-based programming can raise the level of abstraction within a program and is often underutilized [15]. The use of interfaces with the Java programming languages enforces an extra level of indirection and allows the instructors to set a certain level of commonality in a what is to be done manner, without regard to the individualized implementation details. While seeming restrictive on the surface, this still leaves implementation considerations up to individual students and does not impose a common design, just a common interface. This also unifies grading of student work since each individual solution adheres to a prescribed interface, allowing the instructor to save time by constructing the tests a single time and applying them to all students in the course.

This paper describes how we use interfaces in our data structures class, how this enables discrete functional testing of student code, and how this is integrated into the AutoGrader framework. Section 2 describes how interfaces are used in the data structures curriculum. Section 3 gives an overview of the AutoGrader framework, how it is constructed, and how grading packages are written by an instructor. Section 4 places this framework in the context of related work in this field.

\section{INTERFACE-BASED ASSIGNMENTS}


For programming assignments in the context of a data structures course, there is a certain level of commonality between all student projects. When talking about abstract data structures there is a natural tendency towards using a common interface. For instance, data structures for lists can have multiple implementations: array-backed list, singly linked list, and doubly linked list. It doesn't make sense that each of these implementations would present a different interface to the clients of list services. In fact, the usefulness of these data structures increases if they implement common interface[11], emphasizing the flexibility of switching implementations as long as the client only utilizes the interface. Presenting data structures in this fashion is a worthwhile educational objective [15].

We emphasize the use of the Java language construct of interface in order to increase student awareness of component software and to show that we can test software without knowing the implementation details. Black box testing is the process of testing software where we craft tests based solely on the interface of the software, ignoring what we know about the implementation details. For the list assignment in our data structures course, we assign students the task of implementing the standard java.util. List $<E>$ interface [2] by providing both array-backed and linked list implementations. Because of the complexity involved, the subList method is excluded.

Students are also required to write unit tests using JUnit [3] for all aspects of their programs (White box testing). This serves two purposes: (1) to get students to test their code; (2) again, to emphasize the use of interfaces in procedural abstraction, getting them to focus on what is being done, rather than how it is being done. If tests are properly written against the List $\langle\mathrm{E}\rangle$ interface and not directed towards a specific implementation, they are able to reuse their tests for both list implementations, reducing their overall time spent on the assignment.

Configuring a JUnit test class to be able to use multiple implementations can be enabled through the use of an abstract class containing all of the tests, and two sub-classes containing the code to instantiate the list classes. This separation further emphasizes good unit testing in that it makes it more difficult to make one test dependent on another test in the test package. Students are provided with some starter code to demonstrate constructing tests in this way. An excerpt of this starter code is shown in Listings 1 and 2. This example setup works well when students are to provide more than one implementation of the same interface and also provides an example of how code can be tested at the interface level (Listing 1).

Listing 1: GeneralListTest.java

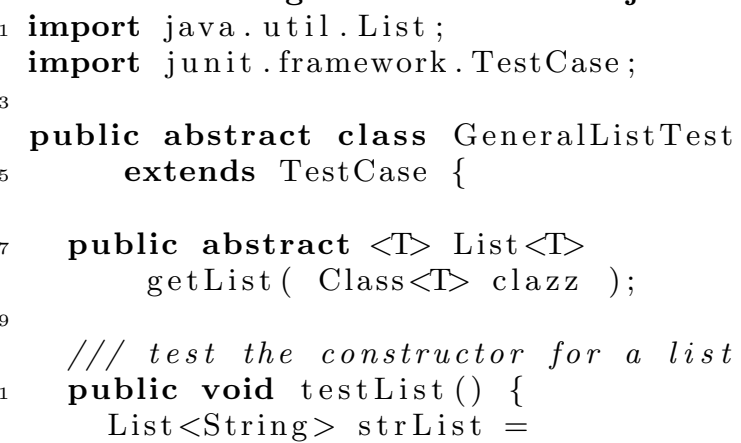

Listing 2: ArrayListTest.java

1 import java.util. List;

import java.util. Random;

public class ArrayListTest extends GeneralListTest \{

7@Override

public $<\mathrm{T}>$ List $<\mathrm{T}>$ getList ( Class $<\mathrm{T}>\mathrm{clazz}) \quad\{$

return new studentid. ArrayList $\angle \mathrm{T}>()$;

11

//return new java.util. ArrayList $<T>()$; \}

$13\}$

The code from Listing 1 is then completed by the student, fully testing the list interface. Listing 2 shows how easy it is to then either test the student developed code (new studentid.ArrayList $\langle\mathrm{T}\rangle())$ or test the built in Java implementation (new java.util. ArrayList $<\mathrm{T}>())$. This enables students to run their tests against an implementation of an object that implements the same interface and is previously known to be in full working order. While the ability to do this is extremely helpful to someone who is new to unit testing, it is not always possible to test an existing implementation. For assignments where there is not a built in data structure, an instructor provided implementation can be used as the reference implementation. On more advanced assignments, students are generally not provided with a working implementation to compare against.

Interfaced-based assignments have been used successfully in our data structures course for lists, binary trees, heaps, sets, maps, and graphs. We emphasize the portability of client code using an interface and thus the ability for a student to write one test suite which can then test multiple implementations. The use of assignments of this type and subsequent automatic grading of student code is not limited to the scope of a data structures course. Any course where the instructor can provide a common interface to students can be handled in this fashion and graded with AutoGrader.

\section{AUTOMATED FUNCTIONAL TESTS}

The AutoGrader framework is based on the concepts and operation of JUnit. It is geared towards the execution of student code where the implementation of a common interface is implemented as described in Section 2. AutoGrader itself is a lightweight framework consisting of only 17 source files and less than 1,500 lines of code, making it easily portable and available for integration with other utilities. In order to automatically grade a student assignment, there must be uniform class names across the assignment. For instance, all students will write a class called ArrayList with different implementations being designated by being included in a package name unique to each student. We 
have found success in having each student create a package whose name is their unique university id, so that classes are named studentid. Arraylist.

An instructor must provide the actual tests to be run, a list of student IDs, and the name of the concrete classes to instantiate. There are three classes in AutoGrader that are used by an instructor when grading an assignment: Graded objects, GradingSession and GradingPackage.

Objects that extend edu.muohio.csa. autograder.framework.Graded are similar to objects that extend TestCase in JUnit. The major difference is that rather than instantiating the object to be tested directly, the object is located using the provided getInstanceOfObject method. An instructor would write an object extending Graded for each objective that they want to test. As in the example described earlier, an object is written to test a specific interface and can even be reused to test different implementations of the same interface for an assignment. Listing 3 shows the beginning of code actually used to exercise student code for the list assignment. Randomization is used within a certain boundary to further exercise the code.

\section{Listing 3: ArrayListTest.java}

1 import java.util. List;

import java.util. Random;

import edu.muohio.csa.autograder. framework. Graded;

import edu.muohio. csa.autograder.

7 framework. GradingException;

9 public class ListGrader extends Graded \{

$11 \quad / / /$ test add

@SuppressWarnings ("unchecked")

public void grade_Add_Element ()

throws GradingException \{

15

int testSize =

(new Random ()). nextInt (100) + 15;

List $<$ Integer $>$ intList $=($ List $<$ Integer $>)$ this . getInstanceOfObject ( List.class);

21

for $($ int $\mathrm{i}=0 ; \mathrm{i}<$ testSize $; \mathrm{i}++)\{$ intList.add ( new Integer(i) ); assertEquals ( new Integer ( $\mathrm{i}+1)$, \} new Integer( intList.size () ) );

$27 \quad\}$

$29\}$

As a matter of convention, any method in a class extending Graded whose name begins with grade will be identified via reflection and considered to be a test method. Several utility methods are included in the Graded object base class to assist with test case construction, including:

1. getInstanceOfObject(class, Object...): Returns an instance of the student code object under test (see example in Listing 3). The actual object instantiated is dependent on the current student being processed in the grading session. The first parameter indicates what class/interface the instantiated object should be returned as. This parameter also determines the return type of the method since getInstanceOfObject is a generic method. Optionally, a variable length list of parameters can be passed, allowing the framework to locate a specific constructor in the student's class using reflection. If the student did not provide an implementation of the required object for this test, that will be logged in the final report.

2. assertEquals ( expected, actual ): An assert equals method that is overloaded for several different types, including Object so that all types of objects can be compared. If the assertion fails, GradingException is thrown and if caught by the framework will be logged in the report for this student.

3. assertTrue() and assertFalse(): Assert that a boolean expression evaluates to true (or false).

4. fail (message): Force this test to be marked as failed immediately. This is useful when you expect code to thrown an exception, and it does not.

This framework would not be workable without the reflection capabilities built into the Java language. Reflection enables the code to be dynamic in nature making use of classes loaders to locate student code, and reflection to actually execute the test methods. To invoke each individual test, a reference to the current Graded object and current test Method are handed to a background invoker. This enables each individual instantiation of student code and execution of tests to be run in a separate thread, allowing for the thread to be terminated should it be found to run for too long. Early versions of AutoGrader did not do this, but infinite loops found in submitted student code required that there be a mechanism to stop testing of a long running method and to move on to other tests and students.

When invoking a test, all possible exceptions are caught and wrapped in a GradingException if the test case itself does not catch the exception. This allows for tests to be constructed that purposely induce exceptions on student code, and records a failure if the exception is not thrown.

After creating the various Graded objects for an assignment, they can be composed into grading packages, and ultimately a grading session. A GradingPackage is constructed by passing in an instance of the Graded object to use, a name to display on the grading output reports, and the name of the student classes to instantiate.

Multiple GradingPackage instances are added to a single GradingSession instance which is also configured with the output directory to use. An optional text user interface can be attached to the grading session which provides constant updates during the execution of the program, but provides a slight performance penalty. Listing 4 shows code actually used to construct the grading session for a programming assignment. This example displays the possibilities for reuse of the same test harnesses to test different implementations of the same interface. Each GradingPackage is a different section in a student's final report, a sample report is available in Appendix A.

Listing 4: GraderMain.java 


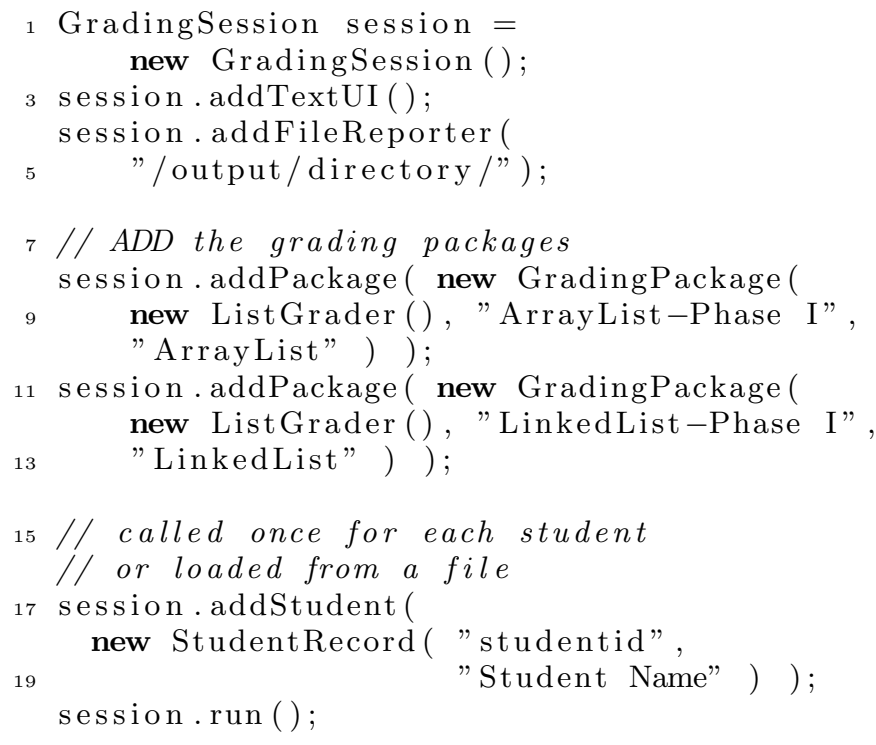

Once the session object's run method is invoked, the tests are run by executing each grading package (in the order added) for each student (in the order added). The appropriate student class file is located using the default class loader, but the object is not yet instantiated. Each test is then run one at a time, with each invocation started in a separate thread and allowed to run up to the configurable timeout value. New instances of the student objects are created each time the getInstanceOfObject method is invoked within a test.

AutoGrader also makes use of PMD [4] to evaluate the style of student code. PMD checks for some common coding problems such as empty catch blocks, empty if statements, comparing objects with $==$ instead of the equals method. A full list of Java style rules supported by PMD is available on the PMD Web site. We have found that PMD provides a good starting point for basic evaluation of coding style, but we still recommend manual evaluation of code by the instructor.

The AutoGrader framework is both powerful and flexible, and integrates with interface-based programming assignments to test student code for functionality and provide feedback reports. For each student assessed during a grading session, a text file is created reporting what the result is for each test. A passed test receives a simple notation of "passed", while a failed test indicates what comparison failed, or which line of code threw an exception during execution. Details of what is being tested are hidden from the student, leaving the option of disclosure to individual instructors. Descriptive test names convey the intent of the test, but we allow students to see the actual code of the tests upon request.

\section{RELATED WORK}

Various efforts for automatic grading of student programming assignments have been undertaken. Many systems rely on pattern matching techniques as is done in both ASSYST [10] and TRY [13]. The major drawback to this approach is only the final output of the program is checked for correctness against the final "correct" output. The AutoGrader framework allows for an instructor to take a narrower view of correctness and assess a student's performance on a method by method basis. Properly constructed test cases will explicitly test a method for correct or incorrect under a single circumstances, leading to the possibility of multiple tests for an individual method.

It has been suggested that introducing named interfaces earlier in the curriculum is worthwhile [15]. Named interfaces help students to learn the concept of procedure abstracting and the decoupling of what an object does from how it is implemented.

Some previous work has been done in testing of student code using the JUnit [3] framework and OCETJ [16]. As with AutoGrader, the authors note that this approach promotes functional testing rather than textual output comparison. OCETJ appears to exercise student code through JUnit, but details of how the testing is implemented and availability of a batch mode are not specified. The AutoGrader framework goes farther in providing a comprehensive framework that can be deployed in batch mode for the instructor and includes background invocations to catch long running student code. The flexibility of the AutoGrader code, including use of the observer pattern [6] makes integrating AutoGrader into your existing submission infrastructure a possibility. Elements of the framework extend the Java Observable class, allowing you to write custom code registering as an observer of grading events.

This work further differentiates itself from use of JUnit in OCETJ in terms of namespaces. If the same JUnit test cases are run on multiple students' code, that requires each student to construct their class in the same namespace. This, in turn, requires a new Java virtual machine instance to test each students code. In AutoGrader, we provide for separate namespaces (Java packages) to be used for each student in the class, allowing the tests to be run in batch mode and in the same Java virtual machine instance.

The AutoGrader framework is more lightweight than the full submission system such as Web-CAT [5]. Our framework is meant to be a solution that can be rapidly deployed and run in an offline fashion or be easily integrated into other online systems. For instance, AutoGrader is integrated with our departmental submission system, but is easily disconnected and can be used with other submission systems. Our solution to automated assessment has the same goals as the Web-CAT system, but takes a different approach.

\section{CONCLUSIONS}

AutoGrader is a framework for Java developed at Miami University for the automatic grading of programming assignments based on common interfaces. This framework has been used successfully for over a year in our data structures course to grade programming assignments for functionality. Grading test suites implemented thus far have ranged in size from 30 to over 100 test cases. Test cases themselves range in complexity in order to adequately test student code. A test suite containing 90 tests can be run for a course containing 50 students in just over 10 minutes, providing an effective use of time for the instructor. Student source code is (optionally) checked for style using PMD [4] and its included style rules.

The assignments are presented to students as a task of implementing an existing or instructor provided interface promoting procedural abstraction while making each student's code testable by the same set of tests. Automated grading of students' code requires an instructor to develop unit tests 
